\title{
Improving Effectiveness of the Active Constellation Extension Method for PAPR Reduction in Generalized Multicarrier Signals
}

\author{
Adrian Kliks · Hanna Bogucka
}

Published online: 16 May 2010

(C) The Author(s) 2010. This article is published with open access at Springerlink.com

\begin{abstract}
In this paper, the Active Constellation Extension (ACE) method of Peak-toAverage Power Ratio (PAPR) reduction is studied for the application in Generalized Mulicarrier (GMC) transmission using nonorthogonal subcarriers. It is shown, that GMC signaling is advantageous for modern wireless communications due to its high spectral efficiency, however, it faces even higher PAPR than in the case of the conventional Orthogonal Frequency Division Multiplexing (OFDM) transmission. The modification of the ACE method for the GMC signals is presented, which takes into account overlapping of data symbol pulses and of their subband spectra. Further improvements of the proposed method are suggested that lead to the decrease of the computational complexity and to more efficacious PAPR reduction in comparison with the conventional ACE scheme. With these improvements, the PAPR reduction for the GMC signaling is the same as for OFDM.
\end{abstract}

Keywords Multicarrier modulation · PAPR reduction - Active constellation extension

\section{Introduction}

Multicarrier (MC) transmission is in the focus of attention of researchers and designers as a suitable technology for fast data transmission in time-dispersive channels (introducing timedispersion of the transmit signal impulses). The Orthogonal Frequency Division Multiplexing (OFDM) is a representative of MC transmission techniques, and is used in both wireless and wired telecommunications. Implementation of the MC modulation can be supported by the Inverse Fast Fourier Transform (IFFT). Recently, MC modulation of non-orthogonal subcarriers, as well as utilization of various pulse shapes in OFDM systems have gained a lot

A. Kliks $(\varangle) \cdot$ H. Bogucka

Chair of Wireless Communications, Poznan University of Technology,

Polanka 3, 60-965 Poznań, Poland

e-mail: akliks@et.put.poznan.pl

URL: www.et.put.poznan.pl

H. Bogucka

e-mail: hbogucka@et.put.poznan.pl 
of attention [1-7]. In this paper, we concentrate on the so-called Generalized Multicarrier (GMC) signaling based on non-orthogonal subcarriers and pulse shaping. The considered transmission scheme offers high spectral efficiency, as it uses densely packed subcarriers, and avoids the application of the guard period. Moreover, application of filtering shapes the subband signal spectrum, so that the out-of-band spectral components decay to zero relatively steeply. This allows to consider GMC technique as very promising for the application in doubly-dispersive channels and in the opportunistic radio, because it minimizes the interference generated by the secondary users to the primary users.

The drawback of the MC signaling is high Peak-to-Average Power Ratio (PAPR). In the presence of nonlinear elements, e.g. power amplifiers, high PAPR results in the in-band distortions, out-of-band radiation, and in the Bit-Error Rate (BER) degradation. Thus, efficient methods to lower the PAPR in MC transmission are required. Numerous techniques, either based on coding, multiple signal representation, constellation modification or signal predistortion have been studied, so far [8]. The Active Constellation Extension (ACE) method of PAPR reduction has been proposed in Refs. [9-11] for the application in OFDM systems. It offers important advantages over other PAPR reduction schemes, as it avoids the necessity to transmit the side information, or to modify the reception algorithm. Moreover, it does not degrade the Bit Error Rate (BER) performance. Finally, it is being proposed for future wireless standards, such as DVB-T2 [12]. Due to its advantageous properties, the ACE method is one of the main candidates for application in future cognitive radios (with an opportunistic spectrum access) that will be based on multicarrier (possibly non-contiguous) signaling. Since the radio-transmission based on non-orthogonal MC waveforms also fits in the requirements yielded to the adaptive and intelligent radios, it is reasonable to adapt ACE method for such systems with GMC signal representation. Thus, here below, we consider its necessary modifications for the GMC signaling. Further refinements of this method are also introduced to increase robustness to nonlinear distortions.

\section{GMC Signals and High Peak Power Problem}

The GMC representation of signals is considered in various publications, however there is no unique definition of such signals. Below, we define the discrete GMC signal $s(n)$ in the same manner as in most of the papers, e.g. as in Refs. [1-3]:

$$
s(n)=\sum_{l \in Z} \sum_{m=0}^{M-1} c_{l m} g_{l m}(n),
$$

where $s(n)(n \in Z)$ is the discrete-time signal belonging to the complex Hilbert space of square-summable sequences $\ell_{2}(Z),\left\{c_{l m}\right\}(l \in Z, m=0, \ldots, M-1)$ are the so-called frame coefficients, here considered as data symbols, $M$ is the number of subcarriers, $Z$ is the set of integers, and $\left\{g_{l m}(n)\right\}$ is a sequence of basis functions, defined as [1-3,13]:

$$
g_{l m}(n)=g(n-l N) \exp [j 2 \pi m(n-l N) / M] .
$$

Above, $N$ is the time spacing between $M$ parallel symbols (in samples) modulating $M$ subcarriers, and $g(n)$ is the pulse shape also called the synthesis window as it is used for the synthesis of signal $s(n)$ as described by (1) Let us note that expression (1) is the Gabor discrete signal expansion (synthesis) of $s(n)$ [1]. In fact, the Gabor-frames theory provides a good tool to analyze GMC signals as we define them [13]. The basis functions $\left\{g_{l m}(n)\right\}$ are also called the Gabor atoms, and constitute the frame if the so-called frame condition holds 
$[13,14]$, i.e. $A\|s(n)\|^{2} \leq\left|\left\langle s(n), g_{l m}(n)\right\rangle\right|^{2}=B\|s(n)\|^{2}, \forall s(n) \in \ell_{2}(Z)$, where $A$ and $B$ are real values called the frame bounds, such that $0<A \leq B<+\infty$. The frame theory is described in sufficient details in [14]. The necessary (but not sufficient) condition for $\left\{g_{l m}(n)\right\}$ to be a Gabor frame is that $N / M \leq 1$. In such a case $g_{l m}(n)$ are sufficiently densely placed in the Time-Frequency (TF) plane, and the dual Gabor frame $\left\{w_{l m}(n)\right\}$ exists $[1,3,13]$, what means that we can inverse formula (1) and calculate the Gabor frame coefficients $\left\{c_{l m}\right\}$ for a given $s(n)$. The frame coefficients $\left\{c_{l m}\right\}$ constitute the TF representation of the signal $s(n)$, therefore this inverse operation is called Gabor-analysis of the $s(n)$ signal, and is defined as:

$$
c_{l m}=\sum_{n \in Z} s(n) w_{l m}^{*}(n),
$$

where $w_{l m}(n)=w(n-l N) \exp [j 2 \pi m(n-l N) / M], w(n)$ is the so-called analysis window (because it is used for the abovementioned analysis), and ( $)^{*}$ denotes complex conjugate. Moreover, (3) describes the Discrete Gabor Transform (DGT). The existence of the dual Gabor frame allows to restore the data symbols at the GMC receiver. Therefore, in the reminder of the paper we will only consider $N / M \leq 1$. Let us note, that if the pulse shape is chosen to be rectangular, and $N=M,\left\{c_{l m}\right\}$ are Fourier coefficients, and GMC modulation becomes OFDM. However-if the window shape is rectangular and $N<M$, overlapping between neighboring pulses exists. Consequently, the OFDM transmission cannot be realized. In a general case however, $g(n)$ is not rectangular, $\left\{g_{l m}(n)\right\}$ are not orthogonal, and overlap both in the time and in the frequency domain. Below in the paper, overcritical sampling, i.e. $N / M<1$, rather than critical sampling, i.e. $N / M=1$, will be considered. (Overcritical sampling condition should not be confused with the non-critical sampling condition of $N / M>1$, e.g. considered in Ref. [6], which is applied to increase the subcarrier spacing). Although it results in a redundant signal expansion, i.e. the number of Gabor coefficients is higher than the number of signal samples, it is usually preferred since the Gabor atoms can be chosen with desirable properties such as good TF localization, which is not possible in the case of critical sampling. Based on the Balian-Low theorem [13], good TF localization of atoms (concentrated around the $(l, m)$ coordinates on the discrete TF plane) results in reduced Inter-Symbol (ISI) and Inter-Carrier interference (ICI), and allows to omit the guard period necessary in the case of OFDM [1-5]. The illustration of the example TF localization of atoms is presented in Fig. 1. An interested reader is referred to [2,3,14-17] for details on Gabor frames theory.

It has been shown in [4] that formula (1) is describing the operation of the DiscreteFourier-Transform synthesis-filter bank, which can be efficiently implemented by the means

Fig. 1 The illustration of overlapping atoms representing the GMC signal on time-frequency plane; $T$-time distance between atoms, $F$-frequency distance between atoms

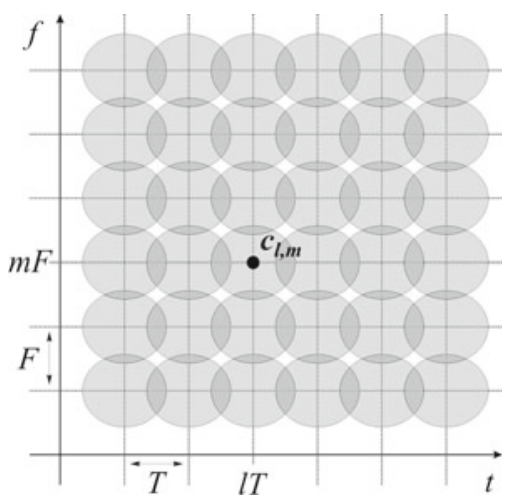


of IFFT and the polyphase filter bank (as long as $M$ is a power of 2, what is the case for our considerations). The polyphase filtering is implemented after IFFT with lower complexity than the pulse-shaping in every GMC subband (at the input of IFFT) [2]. Detailed analysis of the GMC signal generation, as well as its applicability to multi-standard transceivers can be found in Ref.[1-3].

It is worth mentioning that the symbol-based transmission has been assumed in this paper. Thus, one TF-block that consists of $\lambda$ consecutive blocks of $M$-subcarriers corresponds to one GMC-symbol in the time domain appearing at the output of the bank of the polyphase filters. In other words, $\lambda M$ time-frequency data symbols $c_{l m}$ are transformed into one GMC symbol in the similar way as $M$ data symbols in frequency domain are transformed into one time domain symbol in the OFDM case. Let us now consider transmission of random data symbols $c_{l m}$. Thus, the transmit signal $s(n)$ described by (1) is also random. (It has to be noticed that in a case of randomly generated data symbols perfect reconstruction could be impossible $[13,15]$ and some residual interference between neighboring pulses will exist. However, in this paper we do not consider any methods neither for interference cancellation nor for data precoding, which will be suitable for elimination of the effect of this phenomenon. Instead, it is assumed that such method will be applied at the receiver in order to reconstruct the transmit data perfectly.). For the purpose of the comparison of our PAPR reduction methods considered for the GMC scheme with the respective methods proposed for OFDM, let us define the PAPR of the $s(n)$ signal in the same way as it is done for OFDM, i.e. for a block of samples representing $M$ parallel symbols modulating $M$ subcarriers. In case of the GMC modulation this block consists of $N$ samples. Thus, for $n=i N+k, s(n)=s_{i}(k)$, and for the $i$ th block of samples $\mathbf{s}_{i}=\left[s_{i}(0), s_{i}(1), \ldots, s_{i}(N-1)\right]$, PAPR is defined as:

$$
\forall i: \operatorname{PAPR}\left[\mathbf{s}_{i}\right]=\frac{\left\|\mathbf{s}_{i}\right\|_{\infty}^{2}}{E\left\{\left\|\mathbf{s}_{i}\right\|_{2}^{2}\right\} / N},
$$

where $\|\cdot\|_{q}$ denotes the $q$-norm of the enclosed vector, and $E\{\cdot\}$ denotes the expected value. For the GMC signal, the pulse-shaping implemented by the polyphase filtering mentioned above, increases the peak power at the output of the polyphase filter-bank due to the involved convolution of the input signal samples. Thus, depending on the distribution of phases of the input data symbols, GMC signals can have higher instantaneous PAPR and consequently worse PAPR distribution than the OFDM signal. This effect is visible in Figs. 3, 4, 5, and 6, where the experimental Complementary Cumulative Distribution Function (CCDF) of PAPR is presented for both OFDM and GMC signals oversampled by the factor of 4. Overcritical sampling further increases the PAPR value due to the summation of densely overlapping atoms in the GMC modulator.

\section{ACE and its Modification for GMC Signals}

Originally, the idea of the ACE has been presented for the OFDM transmission in Ref. [9]. It is based on the amplitude predistortion of some data symbols at the input of the IFFT (the modulator) to decrease the PAPR at its output. Only the outer constellation symbols can be predistorted in order to keep the constellation minimum distance fixed, what assures no degradation of the BER performance at the receiver. The constellation side-points can be predistorted (moved) only along the half-lines in the outer direction, while the constellation corner-points can be moved in larger quadrant area, as illustrated in Fig. 2 for the 16-QAM signal. 
Fig. 2 ACE-method predistortion options for 16QAM

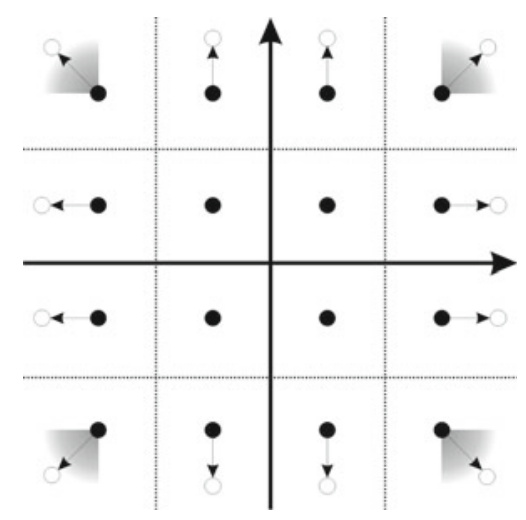

In the ACE algorithm, the set $\mathbf{S}$ of the peaks is determined at the output of the modulator as well as the set $\mathbf{S}_{i d x}$ of their discrete-time indices. A peak is defined as a sample, whose amplitude is higher than the predefined threshold $T_{1}$. The threshold level depends on the Power Amplifier (PA) characteristic, and should be chosen to minimize nonlinear distortions. In Ref. [10,11], it is proposed to compute a metric for every data symbol $c_{m}, 0 \leq m \leq$ $M-1$, and every sample from set $\mathbf{S}$, which reflects the contribution of the $m$ th symbol to the appearance of peaks. Once the metrics are calculated, $L$ constellation outer symbols (either their real, imaginary part or both) of the highest metric values are multiplied by the scaling parameter $\alpha>1$, and the vector of data symbols containing those modified ones modulates the orthogonal subcarriers again.

The drawback of the ACE method is the increase of the transmitted power due to the amplitude amplification of the selected symbols. However, this increase of the signal power depends on the number of predistorted symbols $L$, and the threshold level $T_{1}$, and can be easily controlled by $\alpha$ and $L$. (In Ref. [11] it has been shown that for $L=0.1 M$ and for the considered values of $T_{1}$, the increase of the average signal power does not exceed $0.5 \mathrm{~dB}$, what constitutes $11 \%$ of the original average signal power). It also naturally results in BER performance improvement, even when compared with the transmission not liable to nonlinear distortions. This is because the increase the power of some constellation points causes that they are more robust to errors when compared with the transmission in which ACE is not applied.

Let us summarize pros and cons of the ACE method. Firstly, there is no need to transmit the side information or to modify the receiver structure since only the outer constellation points are modified; in other words, ACE methods ensures backward compatibility with existing transceivers. Then, all of the subcarriers can be used for the transmission of data- or pilot symbols; none of them is devoted for PAPR reduction as in some other methods proposed. Since the minimum distance between the constellation points is not reduced, and the distance between the outer constellation points and the neighboring ones is occasionally increased (at the expense of higher transmit power), the BER of the whole link is not degraded. As it will be shown in the next sections, the amount of the added power as well as the ways of its utilization can be parametrically steered in order to further improve the overall system performance. Due to some power increase of selected pulses on time-frequency plane the amount of out-of-band emission can be also slightly higher when compared with the original signal, since the potential sidelobes of the transmit pulse will carry more energy. However, the in-band average power is also increased in such a case, and the ratio between the in-band 
power and out-of-band power is not degraded. The goal of the ACE method is to minimize the possibility of signal spectrum broadening caused by the presence of non-linear devices. Thus, finally, the application of the ACE method even reduces the level of out-of-band emission comparing with the system where no PAPR reduction methods is applied. It is also worth mentioning that the transmit pulse shapes should be designed in such a way, that the sidelobes of the transmit pulse were below a required power level defined by the spectrum mask.

Application of the ACE method to GMC signals defined in the previous section requires incorporation of the Gabor-atom pulse shape, its duration $N_{g}$ (in samples) and the TF distance between atoms in the metric definition. This is because the GMC-modulated signals interfere with the neighboring (in the TF plane) signals to the degree depending on the pulse shape. The decision concerning predistortion of data symbols should be made for the whole block of symbols consecutively arriving in the considered GMC symbol and modulating the subcarriers (unlike in the case of OFDM and conventional ACE, where this decision can be made for each OFDM symbol separately). Thus, in our case the ACE metric is defined as:

$$
\mu_{l m}=-\sum_{n \in \mathbf{S}_{\mathrm{idx}}} \cos \left(\varphi_{l m n}\right) \cdot|s(n)|^{p},
$$

where $p$ is the parameter of the method, and $\varphi_{l m n}$ is the difference between the phase of the $n$th output peak sample $s(n)$ and the phase of $c_{l m} g_{l m}(n)$, which is the contribution of the input data symbol $c_{l m}$ to this peak. (It is easy to note, that every input data symbol $c_{l m}$ multiplied by $g_{l m}(n)$ forms a component in (1), and thus, contributes to the output sample $s(n)$ ). The metric value indicates to what degree modification of the input symbol $c_{l m}$ can positively affect the decrease of peaks. Note, that in (5) the peak amplitude is taken into account. Let us also note, that $\mathbf{S}$ and $\mathbf{S}_{i d x}$ theoretically contain infinite number of elements (in general $n \in Z$ ). If we consider the symbol based transmission, i.e. the finite range of the time samples, $\mathbf{S}$ contains a number of elements from the whole GMC symbol, unlike in the case of the standard OFDM, when the ACE method can be implemented for distinct OFDM symbols and $n \in[0, \ldots, M-1]$. Moreover, as we can note from (5), in the GMC scheme, the metric is calculated for all symbols transmitted in the GMC time-frequency block, i.e. for all pairs of $(l, m)$ indices while in OFDM the index $l$ does not matter. In practice, the duration of the pulse shape $g(n)$ and atoms $g_{l m}(n)$ is finite, and we may implement (5) for one GMC time domain symbol in a symbol-based transmission [2]. Moreover, overlapping of GMC time-domain symbols can be neglected, if we assume that there is a appropriate gap between consecutive GMC symbols, analogously as the guard period between symbols in the OFDM scheme. It is worth mentioning that by inserting the gap between consecutive GMC symbols we do not destroy the main feature and advantage of GMC transmission, i.e. overlapping of the neighboring atoms in time-frequency domain. Indeed, the neighboring pulses on TF place overlap each other, whereas there is no overlapping between the border pulses of two subsequent GMC time symbols. Let us note that:

$$
\cos \left(\varphi_{l m n}\right)=\operatorname{Re}\left\{\exp \left(j \varphi_{n}\right) \exp \left[-j \varphi_{c_{l m} g_{l m}(n)}\right]\right\},
$$

where $\varphi_{c_{l m} g_{l m}(n)}$ and $\varphi_{n}$ are the phase angles of $c_{l m} g_{l m}(n)$ and $s(n)$ respectively. Moreover, $\exp \left(j \varphi_{n}\right)=s(n) /|s(n)|$ and $\exp \left(-j \varphi_{c_{l m} g_{l m}(n)}\right)=c_{l m}^{*} g_{l m}^{*}(n) /\left|c_{l m} g_{l m}(n)\right|$. Thus, the $\mu_{l m}$ metric for the GMC signals can be calculated as:

$$
\mu_{l m}=\operatorname{Re}\left\{\sum_{n \in \mathbf{S}_{\mathrm{idx}}}-\frac{s(n)}{|s(n)|} \cdot \frac{c_{l m}^{*} g_{l m}^{*}(n)}{\left|c_{l m} g_{l m}(n)\right|}|s(n)|^{p}\right\} .
$$


When the set $\mathbf{S}$ is zero-padded (to achieve the cardinality equal to the order of DGT), we obtain:

$$
\mu_{l m}=-R e\left\{\frac{c_{l m}^{*}}{\left|c_{l m}\right|} D G T_{\text {norm }}^{g^{*}}\left[\tilde{\mathbf{s}} \circ|\tilde{\mathbf{s}}|^{p-1}\right]\right\},
$$

where $D G T_{\text {norm }}^{g^{*}}$ is the operator of the DGT, which uses the following Gabor frame of normalized window functions: $\left\{g_{l m}^{*}(n) /\left|g_{l m}(n)\right|\right\}$ as the analysis frame, $\tilde{\mathbf{s}}$ is the vector of peak samples and zeros padded to positions where peaks do not occur, and "o" denotes the element-wise multiplication of vectors. The metric computation by the means of DGT is relatively low complex because it may use the FFT preceded by the bank of the polyphase filters [2].

\section{ACE Refinements for Enhanced Efficiency}

Below, further modifications of the ACE method are presented, which aim at the enhancement of its performance expressed in terms of the CCDF of PAPR (considered random for the random data symbols).

\subsection{Incorporation of the Near-Threshold Samples (NTS)}

The ACE method can cause the peak re-growth as a consequence of the modification of the constellation shape, particularly, in a GMC transmitter with overlapping symbols. Thus, new peaks can occur, which fall in the nonlinear range of the amplitude input-output characteristic of the PA. We suggest a method to overcome this effect by adding the Near-Threshold time-domain Samples (NTS) to the set $\mathbf{S}$, and by weighting them appropriately:

$$
\mu_{l m}=-\left(\sum_{n \in \mathbf{S}_{\mathrm{idx}}} \cos \left(\varphi_{l m n}\right) \cdot|s(n)|^{p}+\sum_{n \in \mathbf{S}_{\mathrm{NTidx}}} \gamma_{n} \cos \left(\varphi_{l m n}\right)\right)
$$

where $\mathbf{S}_{\mathrm{NTidx}}$ is the set of indices of the near-threshold samples (with amplitudes between the thresholds $T_{1}$ set for the peaks and $T_{2}$ set for the near-threshold samples, $T_{1}>T_{2}$ ), and $\gamma_{n}$ is the weighting function defined as $\gamma_{n}=A \cdot|s(n)|^{p / B}$, i.e. similarly as the weighting function of peaks above $T_{1}$ (equal to $|s(n)|^{p}$ ). The parameters $A$ and $B$ should be tuned to the actual values of $T_{1}$ and $T_{2}$.

\subsection{Flexible Scaling Parameter}

Our idea of further enhancement of the ACE method is to make the scaling parameter $\alpha$ flexible, in such a way that it was an increasing function of $L$ highest metrics:

$$
\forall(l, m) \in \mathbf{S}_{\mathrm{ACE}}: \alpha_{l m}=f_{\alpha}\left(\mu_{l m}\right),
$$

where $\mathbf{S}_{\mathrm{ACE}}$ is the set of pairs of the $(l, m)$ indices, for which $\mu_{l m}$ belongs to the set of $L$ highest metrics. In the same time, we want to ensure that the increase of the transmitted signal power remains unchanged when compared to ACE with constant $\alpha$. For this purpose, let us assume, that the set of possible values of $\alpha_{l m}$ is finite. Moreover, let the sequence of $\alpha_{l m}$ values be the arithmetic progression with the first element equal to 1 and the common difference $\zeta$, so that: 


$$
\forall(l, m) \in \mathbf{S}_{\mathrm{ACE}} \exists ! i \in[1, L]: \alpha_{l m}=\alpha_{i}=1+(i-1) \zeta, c_{l m}=c_{i} .
$$

The value of $\zeta$ can be found by solving the following equation, resulting from the abovementioned constant added-power constraint:

$$
\sum_{(l, m) \in \mathbf{S}_{\mathrm{ACE}}} \alpha^{2} \cdot c_{l m}^{2}=\sum_{i=1}^{L} \alpha_{i}^{2} c_{i}^{2},
$$

where $c_{i}$ is the input symbol (one of the $c_{l m}$ symbols) to be predistorted by $\alpha_{i}$, and $\alpha$ (with no index) is the original constant scaling parameter. By solving (12) and calculating $\alpha_{l m}$ according to (11) we obtain $\alpha_{l m}$ values sorted in the ascending order. We are also able to define the increasing bijective relation between $\alpha_{l m}$ and $\mu_{l m}$, so that the highest value of $\alpha_{l m}$ is applied to the symbol of the highest $\mu_{l m}$, the second highest $\alpha_{l m}$ is mapped to the symbol of the second highest $\mu_{l m}$, and so on. Note, that we have also considered the directly proportional relation between $\alpha_{l m}$ and $\mu_{l m}$ for the scaling parameter flexibility, but in the simulation experiments, the relation $\alpha_{l m}=f_{\alpha}\left(\mu_{l m}\right)$ defined by (11) and (12) has turned to be more effective in PAPR reduction.

\section{Simulation Results and Conclusions}

Below in Figs. 3, 4, 5, and 6, simulation results are presented for the QPSK and 16QAM GMC signal, for which $M>N$ have been chosen (overcritical sampling). The symbol-based transmission has been considered with a number of subcarriers $M=64$, and the number of

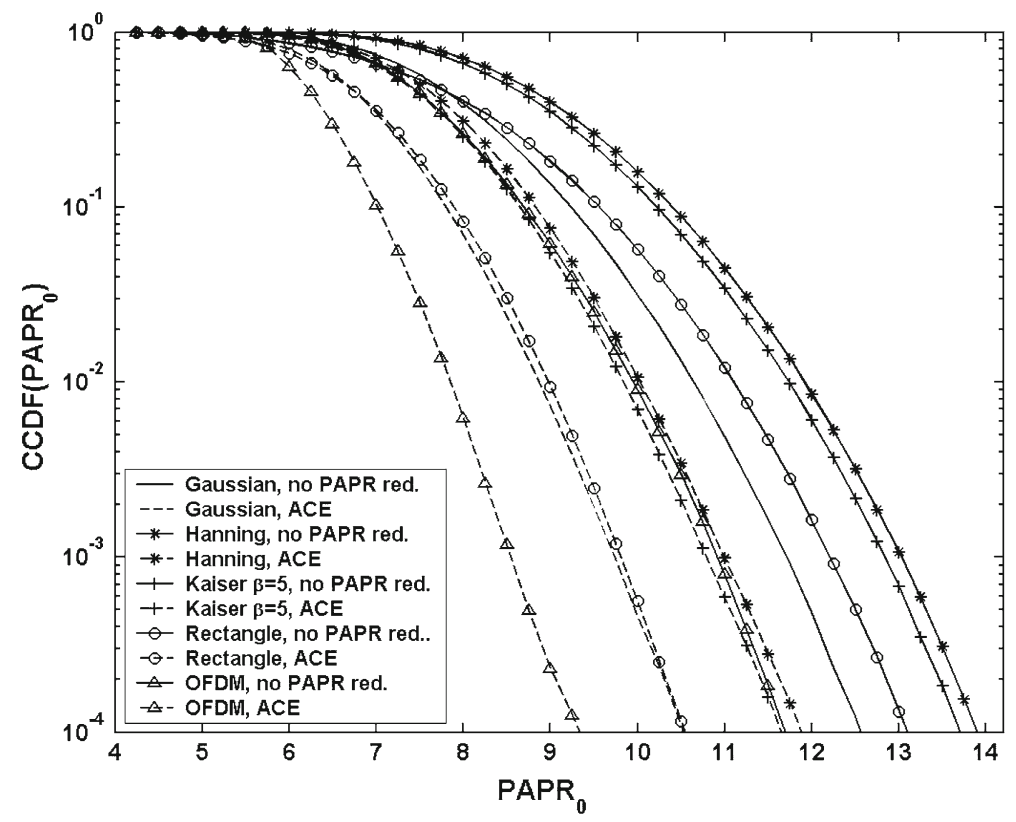

Fig. 3 CCDF(PAPR0) plots obtained for the GMC QPSK transmission for various pulse-shaping windows (i.e. Gaussian, Hanning, Kaiser and rectangular), $M=64, N=48, N g=72$ and for OFDM with no PAPR reduction and applying ACE (constant $\alpha$, no NTS inclusion); $\beta$-Kaiser window parameter 


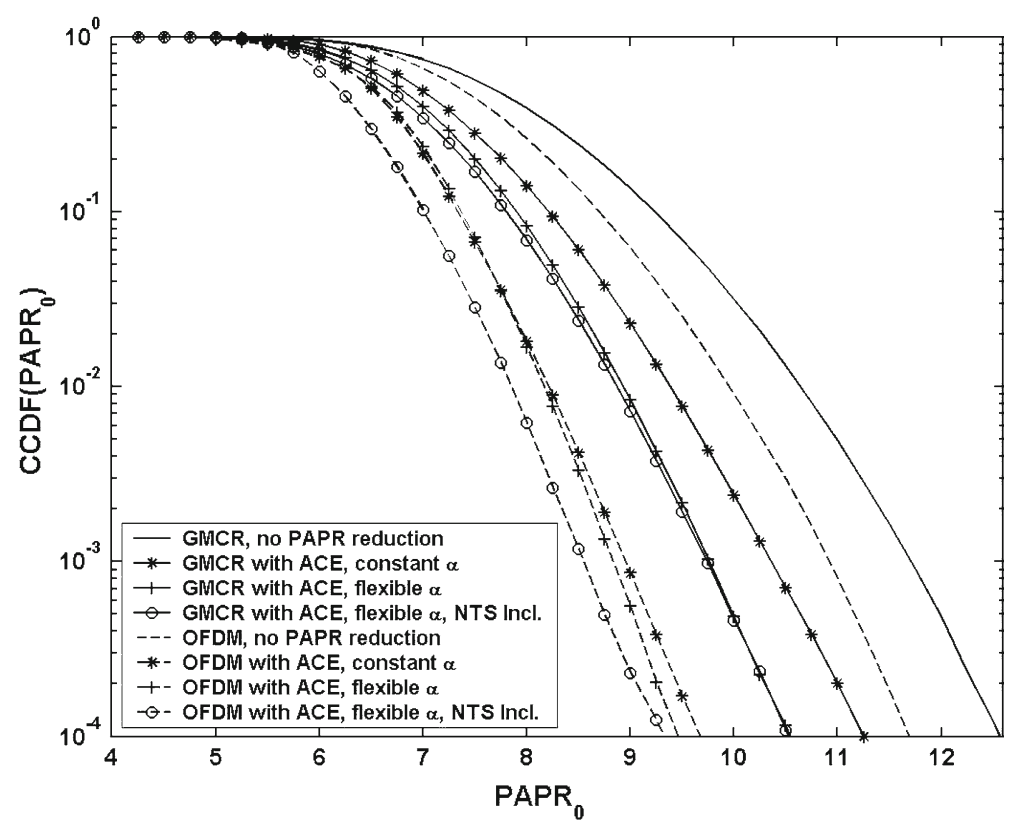

Fig. 4 CCDF(PAPR0) plots obtained for the proposed ACE method improvements for the QPSK-GMC (with Gaussian pulse-shape) and OFDM, $M=64, N=48, N g=72$

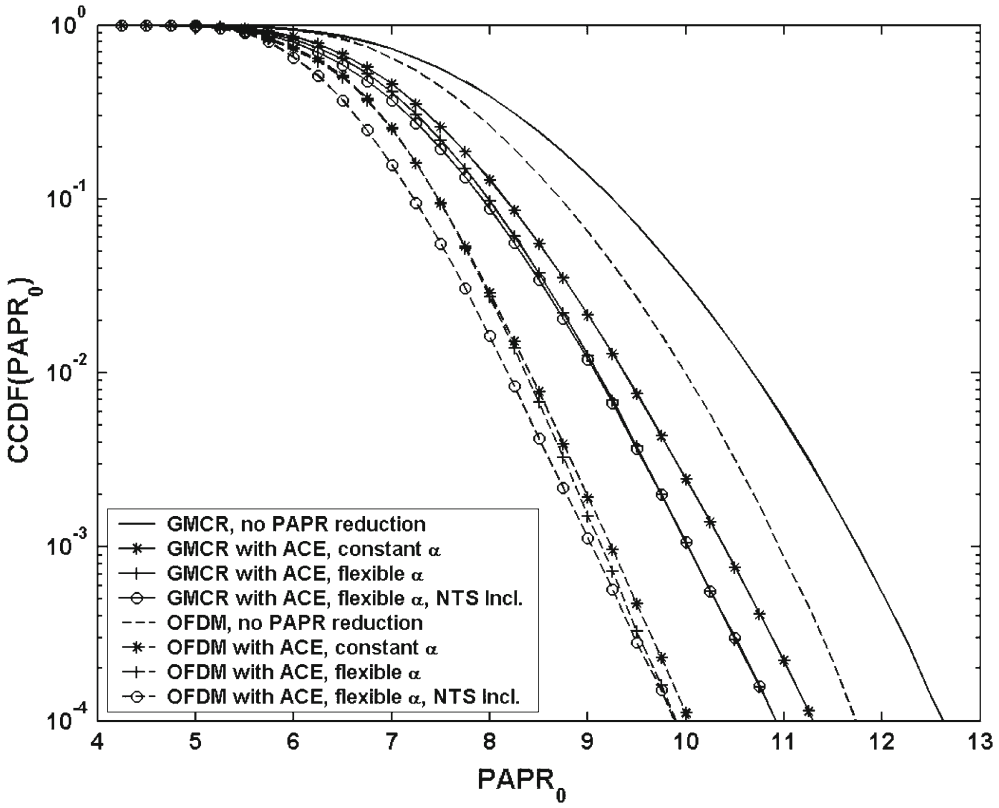

Fig. $5 \mathrm{CCDF}\left(\mathrm{PAPR}_{0}\right)$ plots obtained for the proposed ACE method improvements for the 16-QAM-GMC (with Gaussian pulse-shape) and OFDM, $M=64, N=48, N_{g}=72$ 


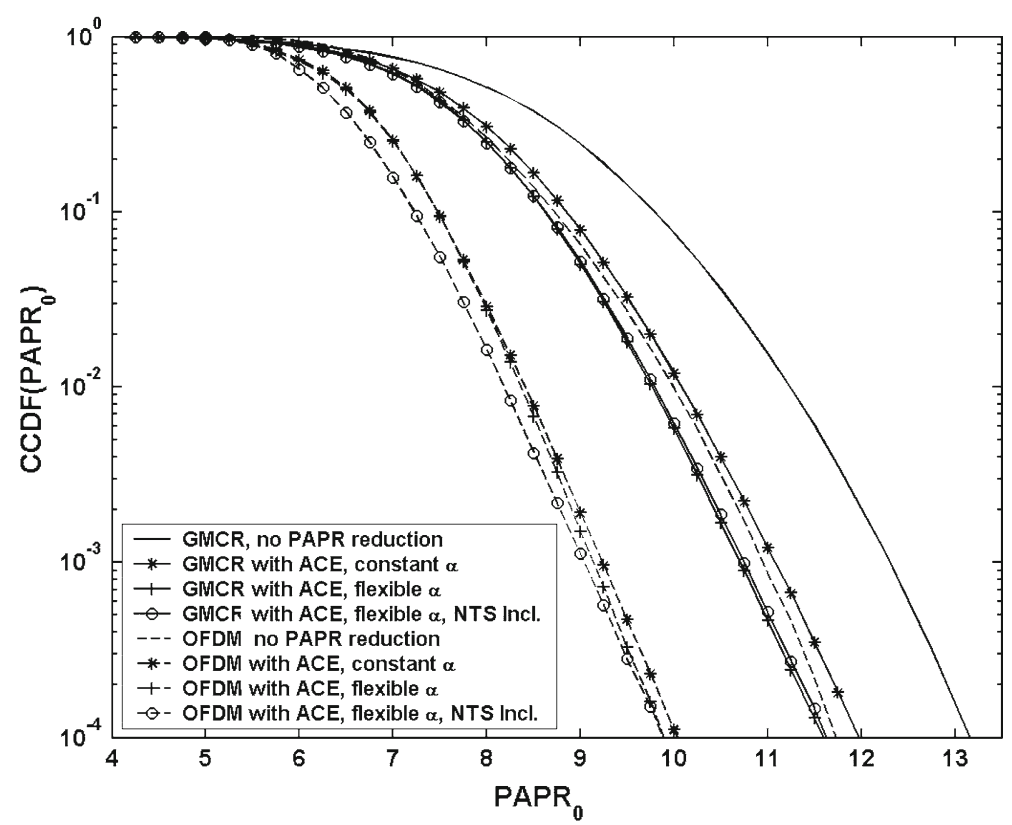

Fig. $6 \mathrm{CCDF}\left(\mathrm{PAPR}_{0}\right)$ plots obtained for the proposed ACE method improvements for the 16-QAM-GMC (with Gaussian pulse-shape) and OFDM, $M=64, N=36, N_{g}=96$

transmitted blocks (of $M$ parallel symbols) equal to 512. (Thus, the total number of symbols in the GMC time-domain symbol equals: $64 \times 512=32,768$.) It has been assumed that subsequent GMC symbols are separated by a guard margin and thus, the signals from consecutive symbols do not overlap. Note, that GMC signaling is characterized by flexibility in the pulse shape selection. Optimization of the pulse shape depends on the optimization criterion, e.g. minimization of ISI or ICI, or other. Figure 3 shows that application of the Gaussian pulse-shape results in the lowest PAPR out of all considered synthesis windows for the GMC transmission. In Figs. 4, 5, and 6, we present simulation results for the Gaussian pulse, which has also good time-frequency localization, what results in minimum ISI and ICI. The duration of this pulse shape is limited to $N_{g}$ samples. The following near-optimal settings of the ACE method have been applied: the number of predistorted symbols $L_{p}=15 \%$ of the number TF data symbols, the $\gamma_{n}$ function (appearing in (9)) parameters: $A=0.5, B=2, T_{2}=0.8$. $T_{1}$, and $T_{1}=3.9 \mathrm{~dB}$ above the signal average power (as in [10]). The relation $\alpha_{l m}=f_{\alpha}\left(\mu_{l m}\right)$ defined by (11) and (12) has been also adopted for the scaling parameter $\alpha$. For comparison, the results obtained for the OFDM with the same number of subcarriers $M$ are presented. The experimental CCDF of PAPR has been estimated for the transmit signals oversampled by the factor of 4 .

One can observe that without the application of any PAPR reduction method, $\mathrm{CCDF}\left(\mathrm{PAPR}_{0}\right)=\operatorname{Pr}\left(\mathrm{PAPR}>\mathrm{PAPR}_{0}\right)$ for a GMC system is much higher than for the relevant OFDM system. Application of the ACE metric modified for the GMC signal (as defined by (5) and (8)) lowers the CCDF(PAPR ${ }_{0}$ ) noticeably. Further ACE refinements (described in Sec. IV) improve the situation even more. The application of flexible $\alpha$ lowers the $\operatorname{CCDF}\left(\mathrm{PAPR}_{0}\right)$ when compared with the results obtained for constant $\alpha$. Additionally, when of the NTS are included in the metric definition, a noticeable decrease of $\operatorname{CCDF}\left(\mathrm{PAPR}_{0}\right)$ is observed for 
OFDM, while for GMC signaling smaller decrease can be noted in the lower range of PAPR 0 values, i.e. below $9 \mathrm{~dB}$ (see "NTS Incl." curves in Figs. 4, 5, and 6).

The above-discussed results for GMC signaling are similar to the ones obtained for OFDM with the original-ACE PAPR reduction (PAPR reduced by approximately $2.5 \mathrm{~dB}$ ), despite much higher initial PAPR inherent for the GMC signal resulting from overcritical sampling and relatively long pulse duration $\left(N_{g}>N\right)$, particularly in the last considered case (Fig. 6).

The method we have presented above together with its modifications and refinements can be applied to any pulse-shaped non-orthogonal multicarrier signal, also with the pulse shape other than Gaussian (Fig. 3). With our proposed improvements of the ACE, the GMC transmission becomes attractive for future modern communication systems, such as opportunistic radio, because its major disadvantage (very high PAPR) is removed.

Open Access This article is distributed under the terms of the Creative Commons Attribution Noncommercial License which permits any noncommercial use, distribution, and reproduction in any medium, provided the original author(s) and source are credited.

\section{References}

1. Kozek, W., \& Molisch, A. F. (1998). Nonorthogonal pulseshapes for multicarrier communications in doubly dispersive channels. IEEE Transactions on Communications, 16(8), 1579-1589.

2. Stefanatos, S., \& Polydoros, A. (2007). Gabor-based waveform generation for parametrically flexible, multi-standard transmitters. In EUSIPCO'07 (pp. 871-875). Poznan, Poland, September 3-7, 2007.

3. Hunziker, T., \& Dahlhaus, D. (2000). Iterative symbol detection for bandwidth efficient nonorthogonal multicarrier transmission. In IEEE vehicular technology conference proceedings, VTC 2000-Spring (pp. 61-65). Tokyo, Japan, May 15-18, 2000.

4. Bolcskei, H., Hlawatsch, F., \& Feichtinger, H. G. (1995). Equivalence of DFT Filter Banks and Gabor Expansions. SPIE Proceedings, 2569, 128-139.

5. Tiejun, W., Proakis, J. G., \& Zeidler, J. R. (2007). Interference analysis of filtered multitone modulation over time-varying frequency-selective fading channels. IEEE Transactions on Communications, 55(4), 717-727.

6. Zhang, J. Q., Kokkeler, A. B. J., \& Smit, G. J. M. (2008). An Oversampled Filter Bank Multicarrier System for Cognitive Radio. IEEE international symposium on personal, indoor and mobile radio communications (PIMRC08) (pp. 1-5). Cannes, France, September 15-18, 2008.

7. Le Khoa, N. (2008). Insights on ICI and its effects on performance of OFDM systems. Digital Signal Processing, 18(6), 876-884.

8. Tellado, J. (2000). Multicarrier modulation with low peak to average power applications to xDSL and broadband wireless. Boston/Dordrecht/London: Kluwer Academic Publishers.

9. Krongold, B. S., \& Jones, D. L. (2003). PAR reduction in OFDM via active constellation extension. IEEE Transactions Broadcast, 49(3), 258-268.

10. Sezinger, S., \& Sari, H. (2006). OFDM peak power reduction with simple amplitude predistortion. IEEE Communications Letters, 10(2), 65-67.

11. Sezginer, S., \& Sari, H. (2007). Metric-based symbol predistortion techniques for peak power reduction in OFDM systems. IEEE Transactions on Wireless Communications, 6(7).

12. DVB BlueBook A122 Rev.1 (01.09) "Frame structure channel coding and modulation for a second generation digital terrestrial television broadcasting system (DVB-T2)".

13. Feichtinger, H. G. \& Strohmer, T. (Eds.). (1998). Gabor analysis algorithms, theory and applications. Basel: Birkhauser.

14. Christensen, O. (2002). An Introduction to Frames and Riesz Bases. Basel: Birkhauser.

15. Qian, S., \& Chen, D. (1996). Joint time-frequency analysis-methods and applications. New Jersey: Prentice Hall Inc.

16. Jung, P., Wunder, G., \& Boche, H. (2002). Comparison of standard OFDM/BFDM to bandwidthefficient pulse shape optimized MC schemes. In 7th international OFDM-workshop (pp. 36-40). Hamburg, Germany.

17. Jung, P., \& Wunder, G. (2007). The WSSUS pulse design problem in multicarrier transmission. IEEE Transactions on Communications, 55(10), 1918-1928. 


\section{Author Biographies}

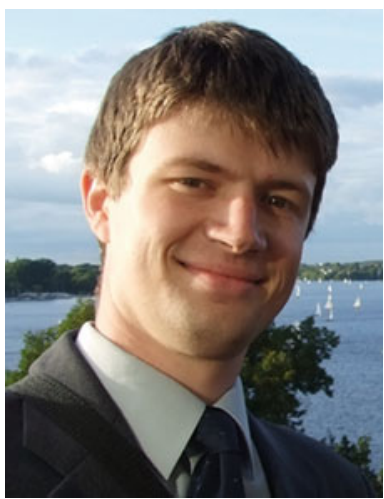

Adrian Kliks received his M.Sc. degree in Telecommunication from Poznan University of Technology in 2005. Since 2005 he has been employed at the Institute of Electronics and Telecommunications, and since 2007 as the senior researcher at the Chair of Wireless Communication in the Faculty of Electronics and Telecommunication. Since 2005 he is the Ph.D. student, and starting form the 1 March 2009 he has been working as the assistant. His research interests cover the wide spectrum of wireless communications. In particular he is interested in multicarrier (both orthogonal an non-orthogonal) systems, in the area of software defined, adaptive and cognitive radios, in hardware implementation on DSP/FPGA and in radio-planning.

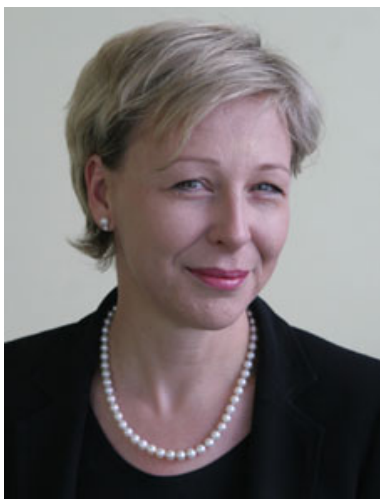

Hanna Bogucka received the M.Sc. and the Ph.D. degrees in Telecommunications from Poznan University of Technology (PUT), Poznan, Poland, in 1988 and 1995 respectively. Since 1988 she has been employed at PUT, currently at the Chair of Wireless Communications as a professor. In 1994, she received the Young Outstanding Scientists Reward from the Polish Science Foundation. Dr. Hanna Bogucka is involved in the research activities in the area of wireless communications, in particular system design and algorithms to provide reliable transmission of digital signals over dispersive radio channels. She was one of the main participants in the scientific projects performed by the Wireless Communications Research Group funded by the State Committee of Scientific Research, European Union 5th, 6th and 7th Framework Programme, and EUREKA research projects, and participated in the consulting for the Polish Telecom (Telekomunikacja Polska S.A.). Dr. Bogucka is the author of more than 70 papers, which have been presented at national and international conferences, and published in leading communication journals. She is also involved in teaching activity at PUT. She gives lectures on radio communications, coding theory and information theory. She has published 3 handbooks in the area of radio communications and digital signal processing. 